\title{
Preface to the special issue on tungsten- and molybdenum-based two-dimensional materials for energy storage and conversion
}

\author{
Yong-Ji Gong ${ }^{1} \cdot$ Zheng Liu $^{2}$
}

Published online: 20 July 2020

(c) The Nonferrous Metals Society of China 2020

Dramatically increased atmospheric $\mathrm{CO}_{2}$ level leads to apparent global climate change, which is an urgent and unpredictable threat to human societies. Developing new energies to replace traditional fossil energy is critical for sustainable development. For example, hydrogen is deemed as a clean and renewable energy alternative to fossil fuels. Alternatively, electricity will play an increasingly critical role in the future in terms of its low cost, diverse sources and wide applications and storage of electric energy is crucial for the applications. Increasing the energy density of lithiumbased batteries is the key to satisfy the continuously growing energy demands for electric vehicles, portable electronic devices and smart grid storage. Specifically, $\mathrm{Li}-\mathrm{S}$ batteries have much higher energy density than that of traditional Liion batteries, which are the most promising energy storage devices in the future.

For these energy storage and conversion techniques, materials are the key to improve the performances. Tungsten, molybdenum and so on based compounds have been proved to be effective catalysts to produce hydrogen or high-performance host for lithium metal anodes or sulfur cathodes. The ultrathin layers of these compounds, two-dimensional (2D) materials, possess advantages of large specific surface area, good conductivity and catalytically active sites. Thus, high-performance catalysts for electrochemical hydrogen evolution reaction and $\mathrm{Li}-\mathrm{S}$ batteries with the high energy density and good rate performance can be achieved using these 2D materials.

Yong-Ji Gong

Yongjigong@buaa.edu.cn

$\triangle$ Zheng Liu

Z.liu@ntu.edu.sg

1 School of Materials Science and Engineering, Beihang University, Beijing 100191, China

2 School of Materials Science and Engineering, Nanyang Technological University, Singapore 637371, Singapore
This special topic focuses on tungsten, molybdenum and other transition metal-based 2D materials for energy storage and conversion. First, a review paper reports the progress on tungsten disulfide-based nanomaterials for energy conversion and storage and some basic characteristics and modification strategies of them are also discussed (Zhong et al.). Then, three papers, including one review paper and two original research articles, focus on the electrocatalytic or photocatalytic performance of $2 \mathrm{D} \mathrm{MoS}_{2}$. Specifically, using single metal atoms doping to active the basal plane of $\mathrm{MoS}_{2}$ for electrochemical hydrogen evolution reaction is briefly reviewed and their mechanism are discussed in depth (Ye et al.). On the other hand, using Au nanoparticles to decorate the surface of $\mathrm{MoS}_{2}$ to improve its activity for hydrogen evolution reaction is proposed in an original research paper (Peng et al.). The much-improved performance can be attributed to the great number of active sites, high conductivity and synergistic effects between Au NPs and $\mathrm{MoS}_{2}$ QDs. In the other research article, Liu et al. systematically studied the relationship between the morphology of $\mathrm{MoS}_{2}$ and its photocatalytic performance, providing strategies to control the morphology and structure of 2D materials to further regulate the photocatalytic performance of $\mathrm{MoS}_{2}$.

Besides, tungsten/molybdenum-based 2D materials also play an important role in $\mathrm{Li}-\mathrm{S}$ batteries. A review paper reports the progress of applications of transition metal sulfides (including $\mathrm{WS}_{2}, \mathrm{MoS}_{2}$ and so on) in the cathode of lithium-sulfur batteries (Gong et al.). Besides sulfide, MXenes, another 2D family containing various transition metals, have been widely applied in electrochemical devices. In this special topic, $\mathrm{Li}$ et al. systematically reviewed the synthesis of MXenes with Mo, $\mathrm{V}$ or $\mathrm{Nb}$ transition metals and their most recent applications are highlighted to provide an outlook for future development. The applications of MXene for sulfur cathodes are summarized in detail (Yang et al.). The different roles MXenes played in sulfur cathodes, including facilitating the electron transfer, tuning the 
adsorption of polysulfides and catalyzing polysulfides conversion, are well discussed.

We hope that this topic could provide a comprehensive insight and inspire innovative ideas to the research area of W-based 2D materials for energy devices, although the present papers cannot entirely cover this broad topic. More contributions in related areas are welcome to be published on the journal Tungsten. At last, we would like to thank all the authors, reviewers, editors and publishing staff for their great efforts to make this issue high-quality and successful.

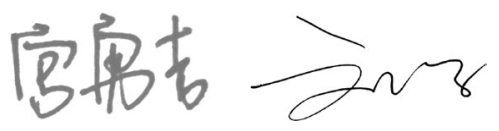

Prof. Yong-Ji Gong

Prof. Zheng Liu

7 July, 2020

Publisher's Note Springer Nature remains neutral with regard to jurisdictional claims in published maps and institutional affiliations. controllable synthesis and properties tuning of two dimensional (2D) materials, 2D alloys and 2D heterostructures for electronics and energy storage and conversion. He has published 100 peer reviewed journal articles, and the work was cited more than 12000 times.

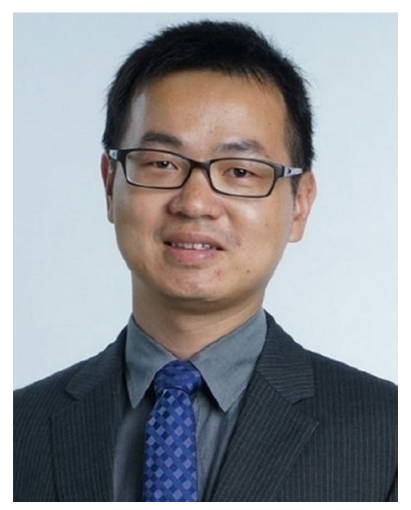

Dr. Zheng Liu is currently an associate professor in School of Materials Science and Engineering, Nanyang Technological University (NTU). He received his B.S. degree (2005) at Nankai University, China, and completed his Ph.D. at National Center for Nanoscience and Technology, China. Before he joined in NTU, he had worked as a postdoctoral fellow (20102012) and research scientist (2012-2013) at Rice University, USA. His research interest is focused on the growth and applications of 2D materials with only single-atom thickness, via innovative methods and artificial intelligence technology. He has published $>300$ peer-reviewed papers including 31 papers in Nature serial journals and Science Advances, 24 in Nano Letters; 30 in Advanced Materials, 19 in ACS Nano, with total citations of $>27000$ and $\mathrm{H}$-index of 77 .

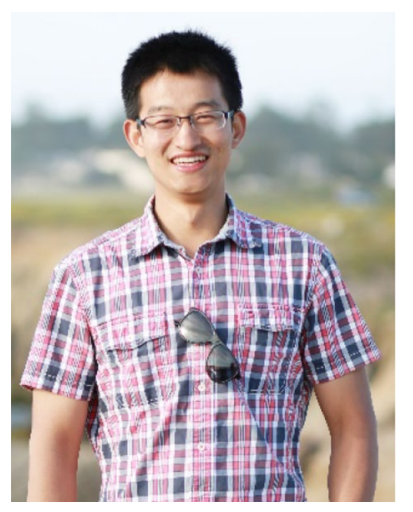

Dr. Yong-Ji Gong is currently a professor in Department of Materials Science and Engineering, Beihang University. He received his B.S. degree (2011) in Chemistry from Peking University and Ph.D. degree (2015) in Chemistry from Rice University. Before he joined in Beihang University, he had worked as postdoctoral fellow in Department of Materials Science and Engineering in Stanford University (2016-2017). His scientific interest is focused on the 